\title{
Ginkgo biloba extract protects human neuroblastoma SH-SY5Y cells against oxidative glutamate toxicity by activating redoxosome-p66Shc
}

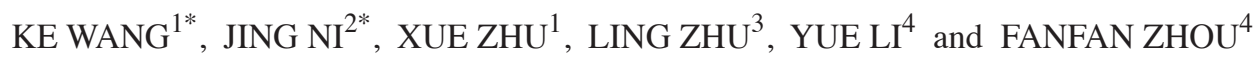 \\ ${ }^{1}$ NHC Key Laboratory of Nuclear Medicine, Jiangsu Key Laboratory of Molecular Nuclear \\ Medicine, Jiangsu Institute of Nuclear Medicine, Wuxi, Jiangsu 214063; ${ }^{2}$ Department of Geriatric \\ Rehabilitation, Jiangsu Rongjun Hospital, Wuxi, Jiangsu 214035, P.R. China; ${ }^{3}$ Save Sight Institute; \\ ${ }^{4}$ Sydney Pharmacy School, The University of Sydney, Sydney, New South Wales 2006, Australia
}

Received June 3, 2020; Accepted December 1, 2020

DOI: $10.3892 /$ etm.2021.10383

\begin{abstract}
Ginkgo biloba extract (GBE), a traditional Chinese herbal medicine component, is widely used to alleviate symptoms of neurodegenerative diseases. It has been confirmed that GBE exerts its pharmacological effect mainly due to its antioxidant activity; however, the molecular mechanism responsible for this effect remains unclear. The aim of the present study was to investigate the detailed mechanism of GBE, the main component of Gingko biloba dropping medicine, against oxidative glutamate toxicity in human neuroblastoma SH-SY5Y cells. The SH-SY5Y cells were untreated or pretreated with GBE followed by glutamate stimulation. Cell viability was assessed using an MTT assay. In addition, oxidative stress indexes, including intracellular ROS generation and NADPH oxidase and caspase activity, were also measured. The protein expression of key signaling factors involved in the redoxosome-p66Shc pathway was evaluated to elucidate the neuroprotective effect of GBE. The results showed that GBE treatment significantly attenuated the glutamate-induced cytotoxicity in SH-SY5Y cells by suppressing oxidative stress. A mechanical study revealed that redoxosome-p66Shc activation was associated with glutamate-induced cytotoxicity,
\end{abstract}

Correspondence to: Professor Ke Wang, NHC Key Laboratory of Nuclear Medicine, Jiangsu Key Laboratory of Molecular Nuclear Medicine, Jiangsu Institute of Nuclear Medicine, 20 Qianrong Road, Wuxi, Jiangsu 214063, P.R. China

E-mail: wangke@jsinm.org

Professor Fanfan Zhou, Sydney Pharmacy School, The University of Sydney, Room N502B, Pharmacy and bank building A15, Camperdown, Sydney, New South Wales 2006, Australia

E-mail: fanfan.zhou@sydney.edu.au

*Contributed equally

Key words: neurodegenerative diseases, Ginkgo biloba extract, glutamate, redoxosome-p66Shc signaling which caused mitochondrial dysfunction and cell death. Interestingly, GBE treatment attenuated the activation of redoxosome-p66Shc in a dose-dependent manner, which suggested that the protective effect of GBE on SH-SY5Y cells against oxidative glutamate toxicity may be mediated by the modulation of redoxosome-p66Shc signaling. The current findings contribute to a better understanding of the therapeutic effect of GBE and indicate that redoxosome-p66Shc signaling might be a novel therapeutic target in the prevention and/or treatment of neurodegenerative diseases.

\section{Introduction}

Neurotransmitters, referred as endogenous chemicals, play important roles in the regulation of brain functions such as memory, mode and behaviors (1). These functions are abnormal when excessive excitatory release of neurotransmitters occurs, leading to neurodegenerative diseases such as Alzheimer's disease, Parkinson's disease and Huntington's disease $(2,3)$. Glutamatergic dysfunction is the key incentive factor to the occurrence and development of specific neurodegenerative diseases (4). A high concentration of glutamate accumulated in the brain resulted in oxidative toxicity and then, neuron death $(5,6)$. The mechanism involved in oxidative glutamate toxicity is complex and largely unknown. Therefore, investigating the molecular mechanism underpinning the neuronal cell damage upon glutamate-associated oxidative stress is highly desired, which contributes to elucidating the pathogenesis of neurodegenerative diseases and developing therapeutic regimens against these diseases.

Ginkgo biloba extract (GBE), the traditional Chinese pharmacopoeia, is used world widely as a herbal medicine against a variety of ailments due to its effect in ameliorating cerebral blood flow as well as antioxidant, anti-inflammatory and antiplatelet properties $(7,8)$. It has been marketed in different formulations, mainly as capsules and Gingko Biloba Dropping pills. It is well known that GBE has neuroprotective effect, which is largely due to its antioxidant activity (9). For instance, literature has showed that GBE exerts its neuroprotective effect via scavenging free radicals and neutralizing ferryl ion-induced 
peroxidation (10). However, the detailed mechanism underpinning the antioxidant activity of GBE against brain nerve cell injury remains unclear. In this study, we explored the neuroprotective effect of GBE against oxidative glutamate toxicity in human neuroblastoma SH-SY5Y cells (11-15), and also investigated the associated molecular mechanism.

\section{Materials and methods}

Chemicals and reagents. GBE was provided by Wanbangde Pharmaceutical Group Co., Ltd. Chemicals such as L-glutamate, MTT (3-(4,5-dimethylthiazol-2-yl)-2,5-dip henyltetrazolium bromide), DCFH-DA and DMSO were obtained from Sigma-Aldrich; Merck KGaA. Antibody were obtained from Santa Cruz Biotechnology,Inc.or Abcam: p-SRC (ab40660, Abcam), SRC (ab109381, Abcam), p-VAV2 (ab86695, Abcam), Vav2 (ab52640, Abcam), p-p66Shc (ab68166, Abcam), p66Shc (ab33770, Abcam), cytochrome $c$ (ab133504, Abcam), $\beta$-actin (sc-58673, Santa Cruz Biotechnology, Inc.), prohibitin (sc-377037, Santa Cruz Biotechnology, Inc.), Goat Anti-Rabbit IgG H\&L (HRP) (ab7090, Abcam), Goat Anti-Mouse IgG H\&L (HRP) (ab205719, Abcam). Rac1 activity assay kit was obtained from Cell Biolabs. Amplex Red hydrogen peroxide/ peroxidase assay kit was obtained from Thermo Fisher Scientific, Inc. Caspase-3 Activity assay kit was obtained from Abcam. Other chemicals and reagents used in this study were obtained from Beyotime.

Cell culture and treatment. Human neuroblastoma SH-SY5Y cells were purchased from the American Type Culture Collection (ATCC). Cells were maintained in culture medium of DMEM (Gibco; Thermo Fisher Scientific, Inc.) containing $10 \%$ fetal bovine serum (FBS, Gibco; Thermo Fisher Scientific, Inc.) with addition of antibiotics $(100 \mathrm{U} / \mathrm{ml}$ penicillin and $100 \mu \mathrm{g} / \mathrm{ml}$ streptomycin, Gibco; Thermo Fisher Scientific, Inc.) at $37^{\circ} \mathrm{C}$ under $5 \% \mathrm{CO}_{2}$. Cells were treated with compounds (Glutamate: $50 \mathrm{mM}$ or GBE: 25,50 and $100 \mu \mathrm{g} / \mathrm{ml}$ ) or transfected with the gene-specific siRNAs (p66Shc: Sense :5'-AUGAGUCUCUGUCAUCGCUTT-3', antisense: 5'-AGC GAUGACAGAGACUCAUTC-3') using Lipofectamine 2000 reagent (Invitrogen; Thermo Fisher Scientific, Inc.) according to the previous study (16).

Cell viability assay. MTT assay was used to determine cell viability. Briefly, cells with or without treatment were incubated with $0.5 \mathrm{mg} / \mathrm{ml}$ MTT solution $(100 \mu \mathrm{l})$ for $3 \mathrm{~h}$ at $37^{\circ} \mathrm{C}$. The culture medium was then replaced with DMSO $(150 \mu 1)$. The absorbance was recorded at a wavelength of $490 \mathrm{~nm}$ using the microstrip reader (Bio-Rad Laboratories).

Oxidative markers analysis. DCFH-DA staining was adopted to assess intracellular ROS generation. Briefly, cells with or without treatment were incubated with DCFH-DA $(10 \mu \mathrm{M})$ for $15 \mathrm{~min}$ at $37^{\circ} \mathrm{C}$. ROS concentration was analyzed using the fluorescence microscope (Leica Microsystems). Intracellular $\mathrm{H}_{2} \mathrm{O}_{2}$ level was measured by Amplex Red assay as previously reported $(17,18)$.

NOX activity analysis. NOX activity was determined by luminescence assay using lucigenin as the electron acceptor generated by the NADPH oxidase complex according to the previous study (19). Cells with or without treatment were sonicated in PBS buffer containing $1 \mathrm{mM} \mathrm{MgCl} 2,1 \mathrm{mM}$ EGTA and protease inhibitors. The lysates $(250 \mu \mathrm{g} / \mathrm{ml}$ of protein) were then incubated with $20 \mu \mathrm{M}$ lucigenin and $100 \mu \mathrm{M}$ NADPH. The emitted luminescence was detected by the luminometer (FluoStar Optima, BMG Labtech). Typically, the readings of the luminescence increased linearly within 5 min and the slope of the trend line was defined as the relative NOX activity.

Cytochrome c release analysis. The cytosol and mitochondrial fractions were prepared for cytochrome $c$ release analysis according to the previous study (20). After treatment, mitochondrial and cytosolic fractions were extracted from the cells using Cell Mitochondria Isolation Kit (Beyotime) according to the manufacturer's instructions. The levels of mitochondrial cytochrome $c$ (Mito Cyto $c$ ) and cytosolic cytochrome $c$ (Cyto Cyto $c$ ) were determined by western blot analysis. $\beta$-actin was used as loading control for cytosolic fraction and prohibitin was used as loading control for mitochondrial fraction.

Western blot analysis. Total protein extract $(20 \mu \mathrm{g})$ from cultured cells was separated by SDS-PAGE and electrophoretically transferred to polyvinylidene fluoride (PVDF) membrane (Beyotime). Target protein was probed by primary antibody $(1: 1,000)$ followed with horseradish peroxidase-conjugated secondary antibody (1:500). The protein signals were detected with chemiluminescence (ECL) detection reagent (Beyotime) and exposed in a dark room. Image $\mathrm{J}$ was used to quantify band densities with normalization to that of $\beta$-actin or prohibitin.

Caspase activity analysis. Caspase activity was assessed using fluorometric assay (Beyotime). Cells with or without treatment were lysed and protein lysate was incubated with caspase-3 substrate DEVD-AFC at $37^{\circ} \mathrm{C}$ for $30 \mathrm{~min}$. The caspase activity was assessed with the spectrofluorometer (Molecular Devices) (excitation wavelength at $400 \mathrm{~nm}$, emission wavelength at $505 \mathrm{~nm})$.

Statistical analysis. Statistical analysis was performed using SPSS 16.0 software package. All data were done in triplicates and the results were from three independent studies. Results of multiple experiments were expressed as means \pm SD. Statistical comparisons were made by Student's t-test between two groups and one-way ANOVA followed by Tukey's post hoc test among multiple groups. $\mathrm{P}<0.05$ was accepted as statistically significant.

\section{Results}

GBE attenuates glutamate-induced cytotoxicity. The SH-SY5Y cells were pre-treatment with or without GBE at a range of concentrations $(0,25,50,100 \mu \mathrm{g} / \mathrm{ml})$ for $24 \mathrm{~h}$, and followed with glutamate incubation $(50 \mathrm{mM}, 6 \mathrm{~h})$. Cell viability was evaluated using MTT assay. Upon GBE pre-treatment (up to $100 \mu \mathrm{g} / \mathrm{ml}$ ), cell viability was not significantly affected, which suggested that GBE is non-toxic to the cells 
A

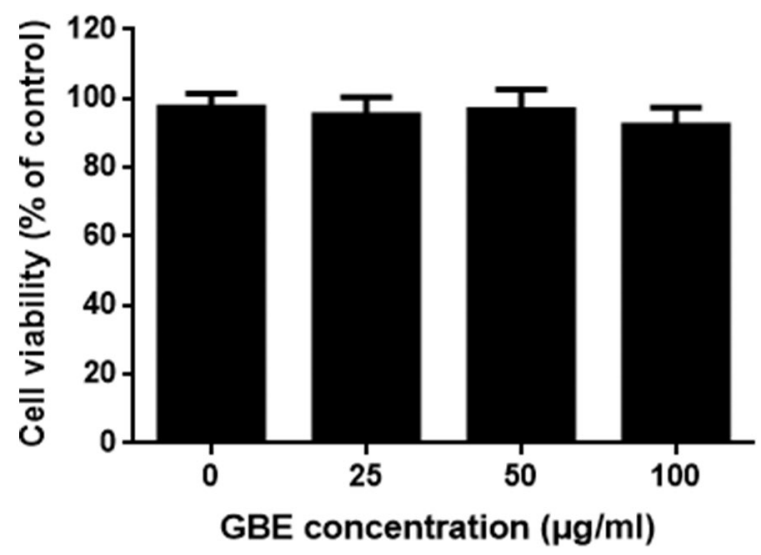

B

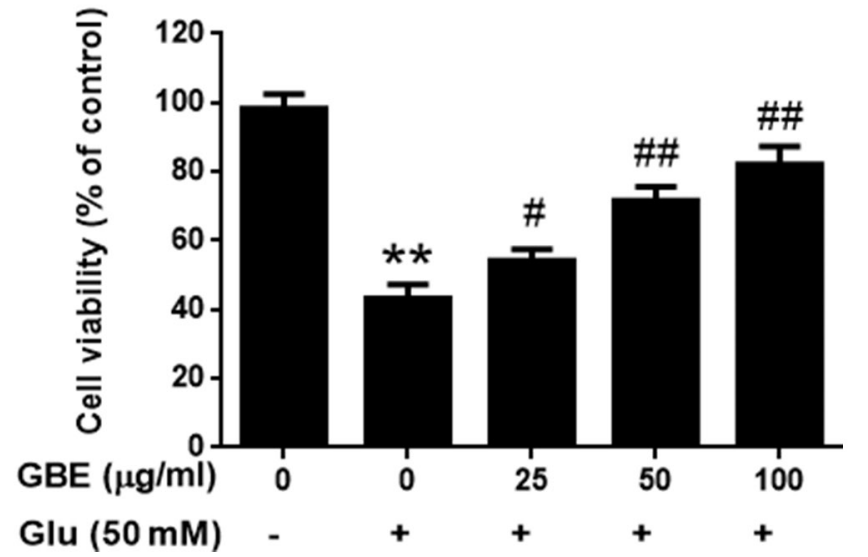

Figure 1. Protective effect of GBE against Glu-induced cytotoxicity in SH-SY5Y cells. (A) Cells were treated with various concentrations of GBE (0, 25, 50, $100 \mu \mathrm{g} / \mathrm{ml}$ ) for $24 \mathrm{~h}$ and cell viability was evaluated using an MTT assay. (B) Cells were pretreated with various concentrations of $\mathrm{GBE}(0,25,50,100 \mu \mathrm{g} / \mathrm{ml})$ for $24 \mathrm{~h}$ and then exposed to Glu $(50 \mathrm{mM})$ for $6 \mathrm{~h}$. After incubation, cell viability was evaluated using an $\mathrm{MTT}$ assay. ${ }^{* *} \mathrm{P}<0.01$ vs. control; ${ }^{\#} \mathrm{P}<0.05$ and ${ }^{\# \#} \mathrm{P}<0.01$ vs. Glu alone. Glu, glutamate; GBE, Ginkgo biloba extract.

A

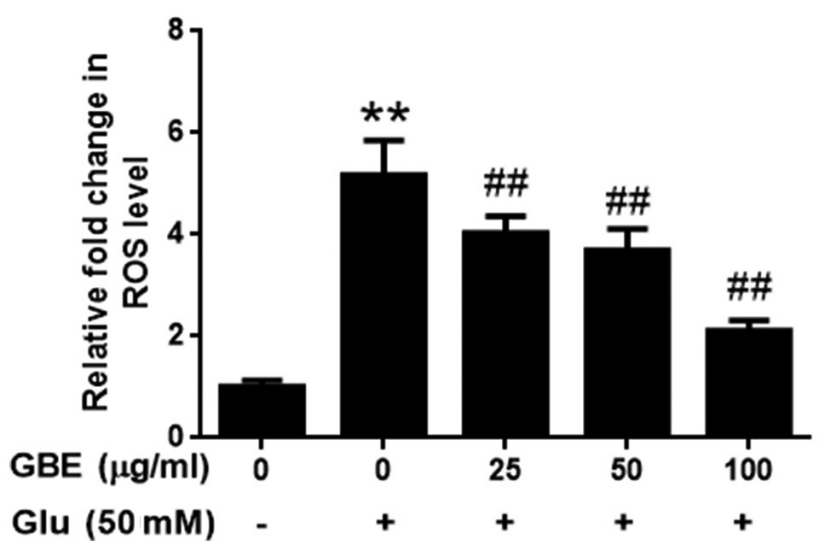

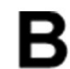

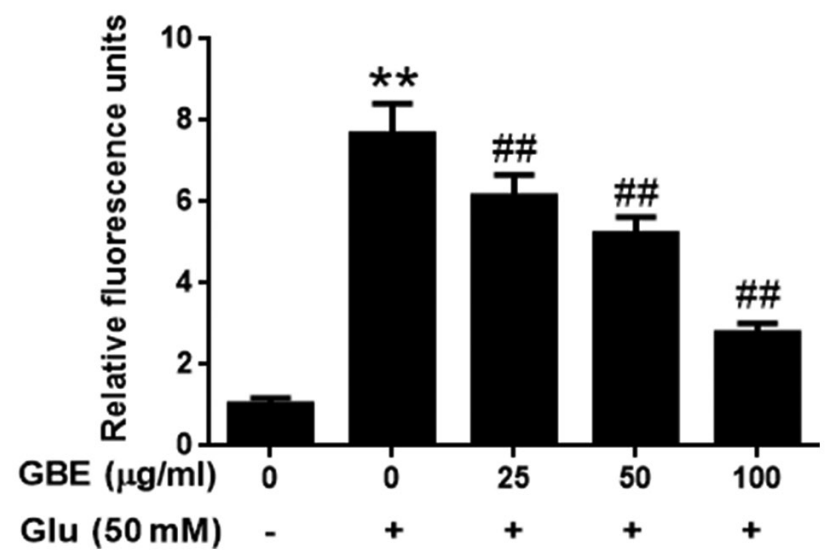

Figure 2. Protective effect of GBE against Glu-induced oxidative injury in SH-SY5Y cells. Cells were pre-treated with various concentrations of GBE $(0,25$, $50,100 \mu \mathrm{g} / \mathrm{ml})$ for $24 \mathrm{~h}$ and then exposed to Glu $\left(50 \mathrm{mM}\right.$ ) for $2 \mathrm{~h}$. (A) ROS level was evaluated using CM-H2DCFDA. (B) $\mathrm{H}_{2} \mathrm{O}_{2}$ level was evaluated using an Amplex Red assay. ${ }^{* *} \mathrm{P}<0.01$ vs. control; ${ }^{\# \#} \mathrm{P}<0.01$ vs. Glu alone. Glu, glutamate; GBE, Ginkgo biloba extract; ROS, reactive oxygen species.

(Fig. 1A). Comparing the groups treated with glutamate, GBE dose-dependently attenuated the glutamate-induced cytotoxicity (Fig. 1B). Such findings demonstrated the protective effect of GBE against glutamate-induced toxicity in SH-SY5Y cells.

GBE attenuates glutamate-induced oxidative injury. The effect of GBE against glutamate-induced oxidative injury in SH-SY5Y cells was further evaluated with the measurement of ROS and $\mathrm{H}_{2} \mathrm{O}_{2}$. As shown in Fig. 2, exposure of cells to glutamate $(50 \mathrm{mM})$ for $2 \mathrm{~h}$ resulted in the significantly upregulated levels of intracellular ROS and $\mathrm{H}_{2} \mathrm{O}_{2}$, potentiating the oxidative toxicity of glutamate. However, cells pre-treated with GBE ( 0 , $25,50,100 \mu \mathrm{g} / \mathrm{ml}, 24 \mathrm{~h}$ ) prior to glutamate incubation resulted in a dose-dependent attenuation of ROS and $\mathrm{H}_{2} \mathrm{O}_{2}$ generation (Fig. 2). These results indicated that GBE exerted its protective effect against glutamate-induced toxicity by attenuating oxidative stress in SH-SY5Y cells.
GBE attenuates glutamate-induced redoxosome activation. To examine the molecular mechanism responsible for the neuroprotective effect of GBE, redoxosome activity was measured. As shown in Fig. 3, glutamate treatment $(50 \mathrm{mM}$, $2 \mathrm{~h}$ ) induced the upregulated phosphorylation of Src tyrosine and Vav2 tyrosine, which in turn induced the increased expression of active Rac1-GTP, leading to NADPH oxidase (NOX) activation in the Rac1-GTP-dependent manner. However, the incubation of Rac inhibitor (NSC23766, $80 \mu \mathrm{M}, 6 \mathrm{~h}$ ) or NOX inhibitor (VAS2870, $10 \mu \mathrm{M}, 6 \mathrm{~h}$ ) potently protected the cells from the cytotoxic effect of glutamate (Fig. 4). Furthermore, the pre-treatment of $\operatorname{GBE}(0,25,50,100 \mu \mathrm{g} / \mathrm{ml}, 24 \mathrm{~h})$ prior to glutamate incubation $(50 \mathrm{mM}, 6 \mathrm{~h})$ resulted in a dose-dependent attenuation of redoxosome activation.

GBE inhibits redoxosome-dependent p66Shc activation. To investigate whether glutamate-induced redoxosome activation 
A

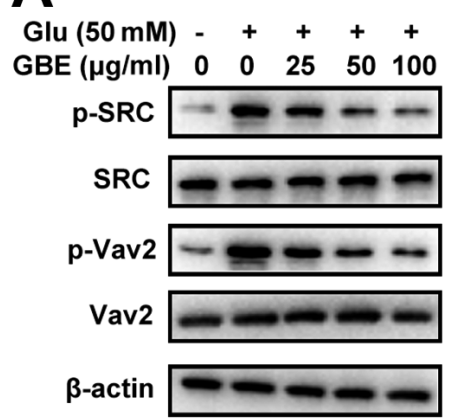

B

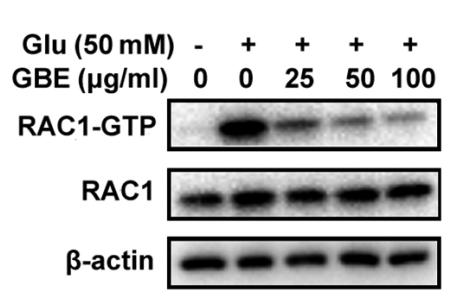

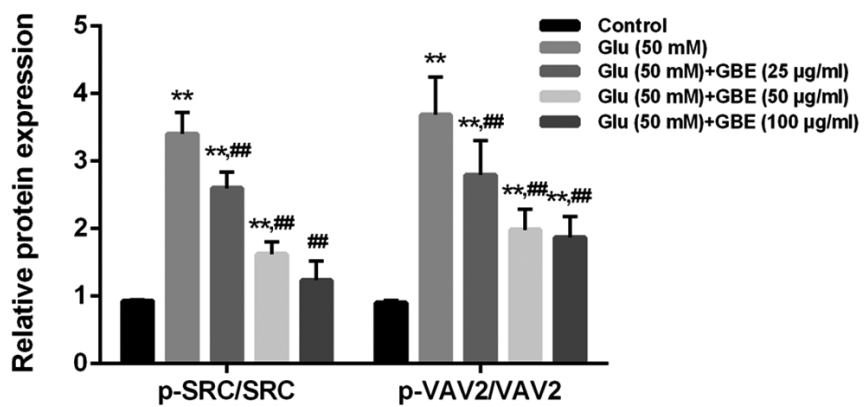

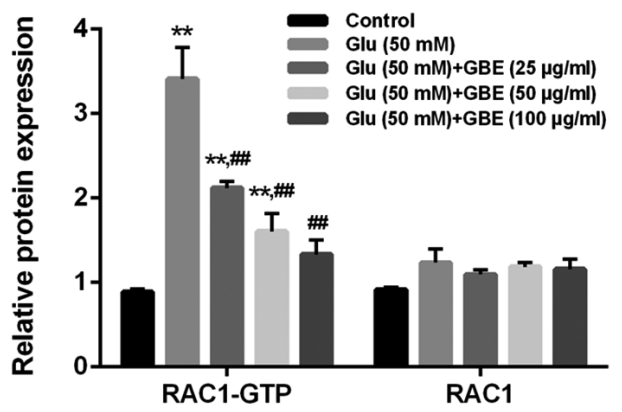

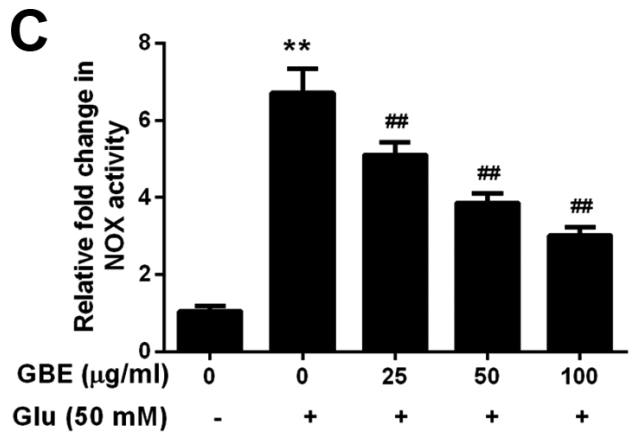

Figure 3. Protective effect of GBE against Glu-induced redoxosome activation in SH-SY5Y cells. Cells were pretreated with various concentrations of GBE $(0,25,50,100 \mu \mathrm{g} / \mathrm{ml})$ for $24 \mathrm{~h}$ and then exposed to glutamate $(50 \mathrm{mM})$ for $2 \mathrm{~h}$. (A) Protein levels of p-SRC, SRC, p-Vav2 and Vav2 were evaluated by western blot analysis. (B) Representative Rac1 western blot is shown. (C) NADPH oxidase activity was measured. ${ }^{* *} \mathrm{P}<0.01$ vs. control; ${ }^{* \#} \mathrm{P}<0.01 \mathrm{vs}$. Glu alone. Glu, glutamate; GBE, Ginkgo biloba extract; p, phosphorylated; SRC, proto-oncogene tyrosine-protein kinase Src; Vav2, guanine nucleotide exchange factor VAV2.

A

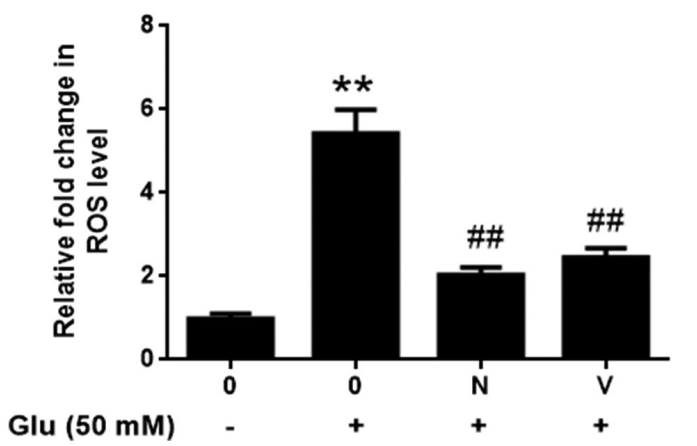

B

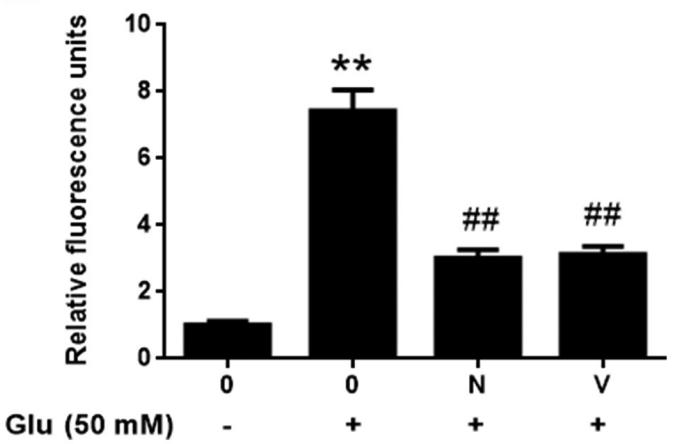

Figure 4. Effect of redoxosome inhibitors against glutamate-induced oxidative stress in SH-SY5Y cells. Cells were pretreated with Rac inhibitor N ( $80 \mu \mathrm{M})$ or NADPH oxidase inhibitor $\mathrm{V}(10 \mu \mathrm{M})$ for $6 \mathrm{~h}$ and then exposed to Glu $(50 \mathrm{mM})$ for $2 \mathrm{~h}$. (A) ROS level was evaluated using CM-H2DCFDA. (B) $\mathrm{H}_{2} \mathrm{O}_{2}$ level was evaluated using an Amplex Red assay. ${ }^{* *} \mathrm{P}<0.01$ vs. control; ${ }^{\#} \mathrm{P}<0.01$ vs. Glu alone. N, NSC23766; V, VAS2870; Glu, glutamate; ROS, reactive oxygen species.

impacts on p66Shc activity, the expressions of p-p66Shc and distribution of p66Shc were measured with or without GBE pre-treatment. As shown in the Fig. 5, glutamate treatment (50 mM, $2 \mathrm{~h}$ ) induced p66Shc serine36 phosphorylation and mitochondrial translocation of p66Shc. However, Rac inhibitor (NSC23766, $80 \mu \mathrm{M}, 6 \mathrm{~h}$ ) or NOX inhibitor (VAS2870, $10 \mu \mathrm{M}$, $6 \mathrm{~h})$ incubation blocked the effect of glutamate on p66Shc activation. Moreover, cells pre-treated with $\operatorname{GBE}(0,25,50$, $100 \mu \mathrm{g} / \mathrm{ml}, 24 \mathrm{~h})$ prior to glutamate incubation $(50 \mathrm{mM}, 2 \mathrm{~h})$ led to a dose-dependent attenuation of p66Shc activation (Fig. 6).

GBE attenuates glutamate-induced mitochondrial dysfunction. Mitochondria translocation of p66Shc induces mitochondrial dysfunction. Thus, we also assessed the mitochondrial function in cells with or without GBE pre-treatment. As shown in the Fig. 7, glutamate treatment $(50 \mathrm{mM}, 6 \mathrm{~h})$ resulted in the reduced mitochondrial membrane potential (MMP), increased cytochrome $c$ release and activation of caspase-3. However, p66Shc inhibition blocked glutamate-induced mitochondrial dysfunction. Furthermore, cells pre-treated with $\operatorname{GBE}(0,25,50,100 \mu \mathrm{g} / \mathrm{ml}, 24 \mathrm{~h})$ prior to glutamate incubation $(50 \mathrm{mM}, 6 \mathrm{~h})$ showed the decreased mitochondrial dysfunction dose-dependently (Fig. 8).

\section{Discussion}

Gingko biloba extract (maidenhair tree) is a traditional Chinese medicine, which has been clinically adopted in the 

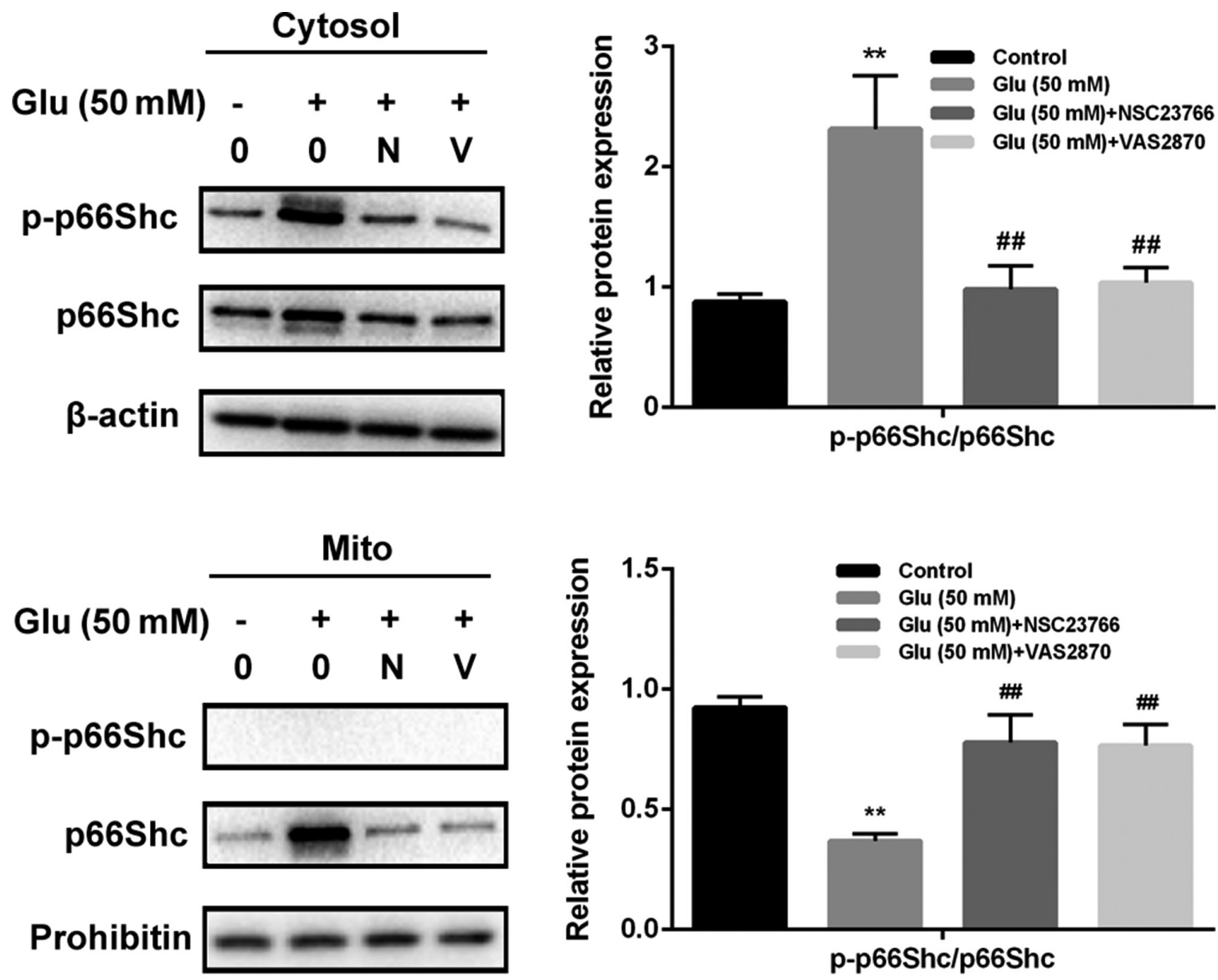

Figure 5. Role of p66Shc in glutamate-induced redoxosome activation in SH-SY5Y cells. Cells were pretreated with Rac inhibitor NSC23766 (80 $\mu \mathrm{M})$ or NADPH oxidase inhibitor VAS2870 $(10 \mu \mathrm{M})$ for $6 \mathrm{~h}$ and then exposed to Glu $(50 \mathrm{mM})$ for $2 \mathrm{~h}$. The protein levels and distribution of p-p66Shc and p66Shc were evaluated by western blot analysis. ${ }^{* *} \mathrm{P}<0.01$ vs. control; ${ }^{\# \#} \mathrm{P}<0.01$ vs. Glu alone. N, NSC23766; V, VAS2870; p, phosphorylated; Glu, glutamate; Mito, mitochondrial fraction.

treatment of various neurodegenerative diseases for hundreds of years (21). For example, EGb761, one of the standard extracts isolated from Ginkgo biloba leaves, has been used in treating neurological diseases including Alzheimer's disease, Parkinson's disease and Huntington's disease $(22,23)$. GBE of Gingko Biloba Dropping is another standard exact, which is prepared through alcohol extraction and then purification with a macroporous resin column. Considering national pharmacopoeia standards, the quality indexes of GBE are quite standardized: Flavonoid glycosides $\geq 24 \%$, terpene lactones $\geq 6 \%$, ginkgolic acids $\leq 5$ or $10 \mathrm{ppm}$. The most important flavonoids of GBE are glycosides of kaempferol, quercetin, and isorhamnetin with glucose or rhamnose. Ginkgolides, only present in Ginkgo biloba extract but any other living species, can be divided into types A, B, C, and J (a very small quantity). Its other clinically important ingredients are not well studied, such as procyanidins, catechins and organic acids (24).

It is well known that GBE has potent neuroprotective effect, which is largely due to its anti-oxidant activity. It can alleviate ischemia, oxidative stress and $\beta$-amyloid-induced toxicity (25). Huang et al have reported that EGb761 can protect SH-SY5Y neuroblastoma cells against glutamate toxicity via inhibiting excitotoxicity and calcium influx as well as reducing the expression of apoptotic markers (26). However, little is known about
GBE. In this study, we investigated the anti-oxidative effect of GBE, the main active extract from the Ginkgo biloba dropping pills, against glutamate-induced toxicity in SH-SY-5Y cells. We found that GBE has comparable effect to that of EGb761 (data not shown). More importantly, we investigated the molecular mechanism of GBE against glutamate-induced oxidative toxicity. According to the previous studies, $50 \mathrm{mM}$ of glutamate is effective to induce oxidative toxicity in SH-SY5Y cells; while $0-100 \mu \mathrm{g} / \mathrm{ml}$ of GBE was selected as the desired concentration range to test for its anti-oxidative effect (27-29).

Redox signaling is a key player in the regulation of physiological processes (30). Accumulating evidence reveals that the abnormality of redox signaling in response to stress leads to the occurrence and development of neurological disorders and other diseases $(31,32)$. Redoxosome is a fledgling area of cellular signaling through superoxide-producing endosomes, which acts via specific redox modifications on numerous proteins and enzymes (33). Redoxosome activation includes Src kinase-dependent Vav2 tyrosine phosphorylation, Rac1-GTP activation and activation of NADPH oxidase (34). In this study, glutamate treatment remarkably induced oxidative stress by activating redoxosome signaling, which is associated with Src-VAV2-Rac1-NOX activation; while Rac inhibitor (NSC23766) or NOX inhibitor (VAS2870) could potently 

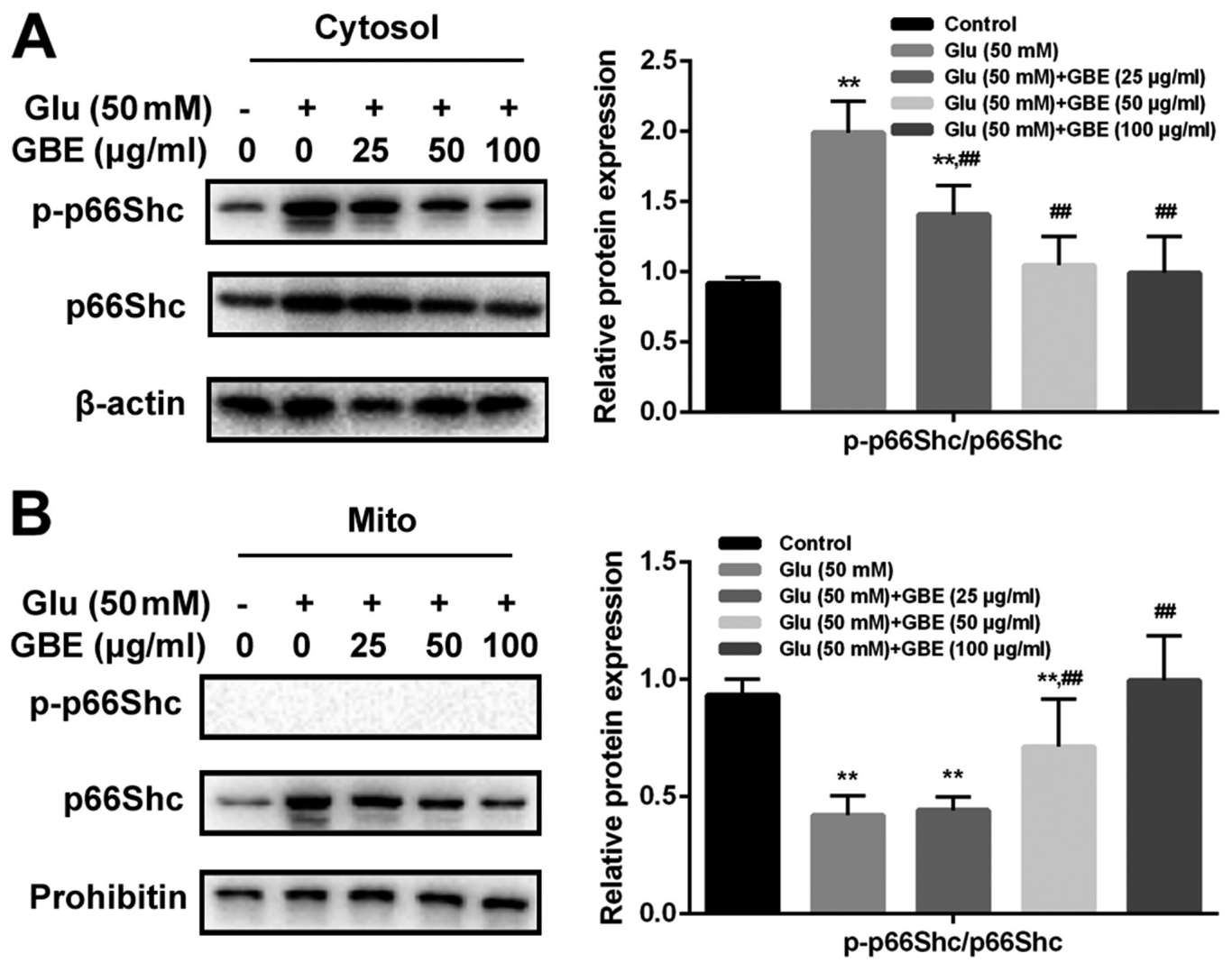

Figure 6. Protective effect of GBE against Glu-induced p66Shc activation in SH-SY5Y cells. Cells were pretreated with various concentrations of GBE (0, 25, $50,100 \mu \mathrm{g} / \mathrm{ml})$ for $24 \mathrm{~h}$ and then exposed to $\mathrm{Glu}(50 \mathrm{mM})$ for $2 \mathrm{~h}$. The protein levels and distribution of p-p66Shc and p66Shc in the (A) cytosolic fraction and (B) mitochondrial fraction were evaluated by western blot analysis. ${ }^{* *} \mathrm{P}<0.01$ vs. control; ${ }^{\# \#} \mathrm{P}<0.01$ vs. Glu alone. Glu, glutamate; GBE, Ginkgo biloba extract; Mito, mitochondrial fraction; p, phosphorylated.

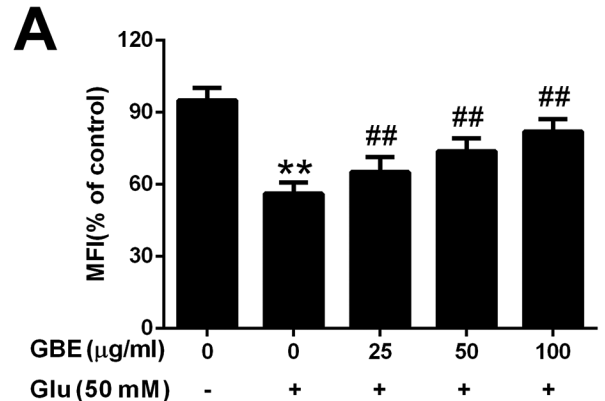

C.

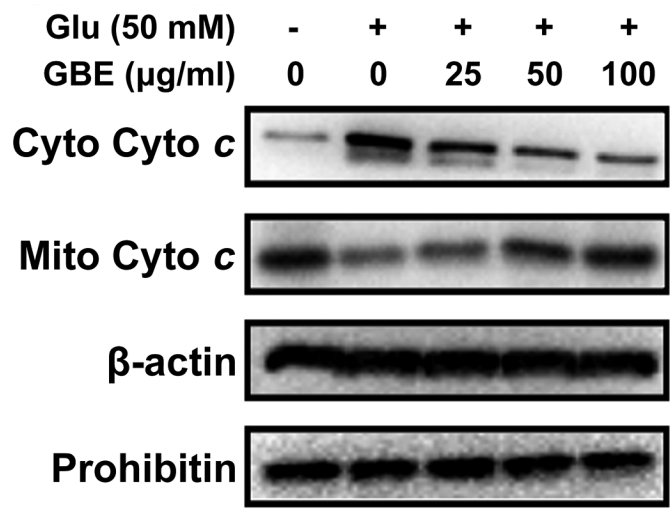

B
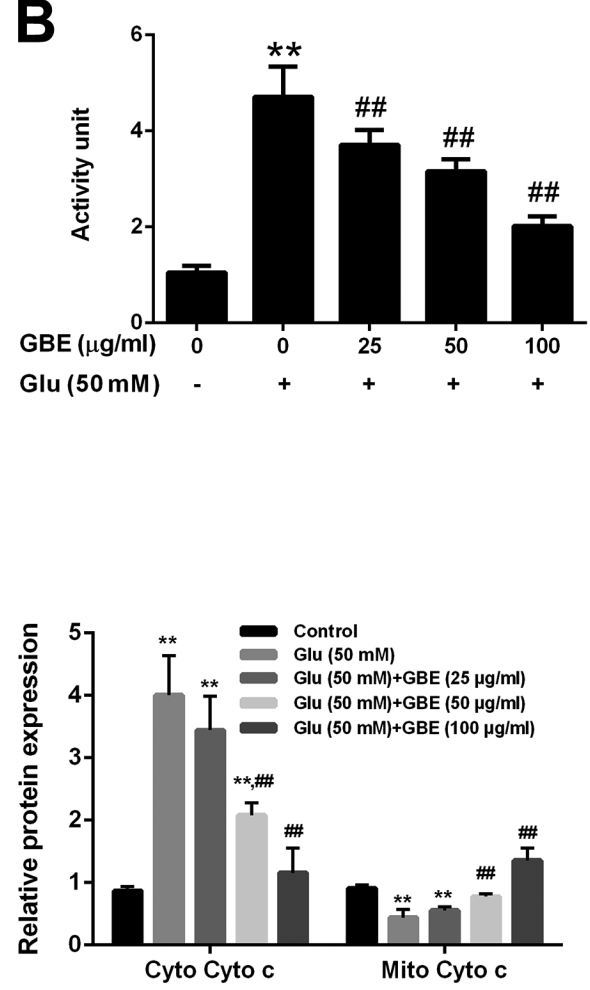

Figure 7. Protective effect of GBE against glutamate-induced mitochondrial function in SH-SY5Y cells. Cells were pretreated with various concentrations of $\operatorname{GBE}(0,25,50,100 \mu \mathrm{g} / \mathrm{ml})$ for $24 \mathrm{~h}$ and then exposed to Glu $(50 \mathrm{mM})$ for $6 \mathrm{~h}$. (A) MFI of Rh123. (B) Caspase-3 activity. (C) Mito Cyto $c$ and Cyto Cyto $c$ levels were determined by western blot analysis. ${ }^{* *} \mathrm{P}<0.01$ vs. control; ${ }^{\sharp \#} \mathrm{P}<0.01$ vs. Glu alone. Glu, glutamate; GBE, Ginkgo biloba extract; MFI, mean fluorescence intensity; Mito Cyto $c$, mitochondrial cytochrome $c$; Cyto Cyto $c$, cytosolic cytochrome $c$. 
A

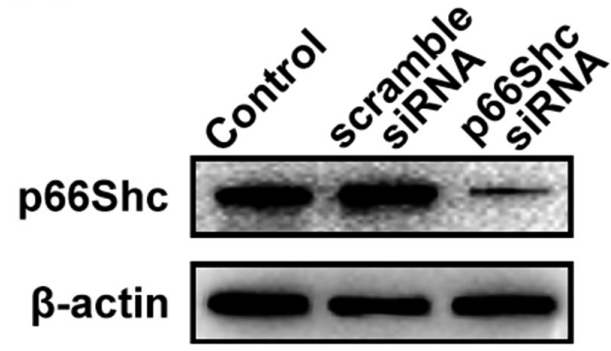

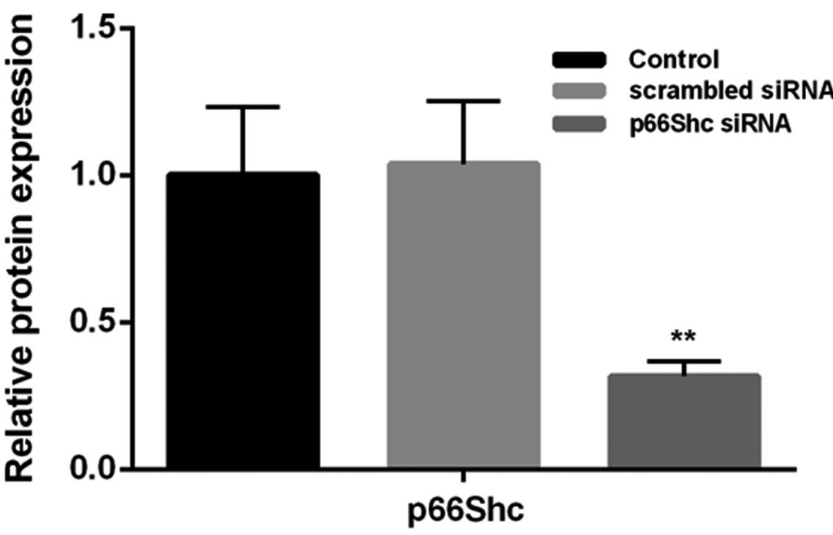

C

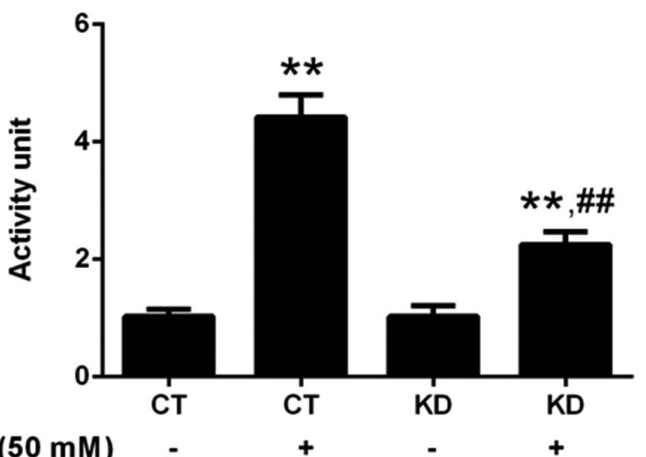

Glu (50 $\mathrm{mM})$ -

D

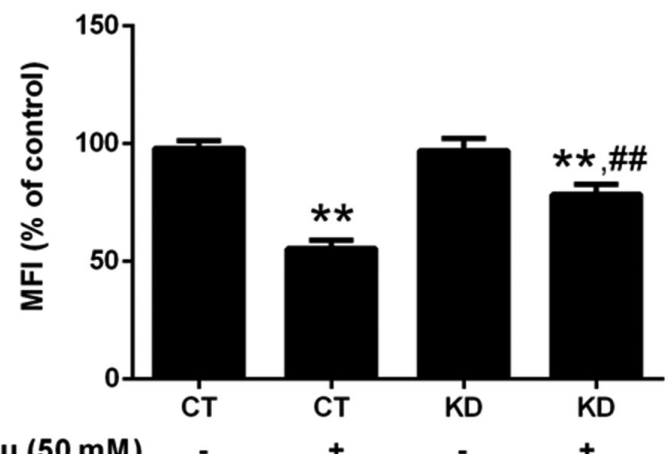

Glu (50 mM)
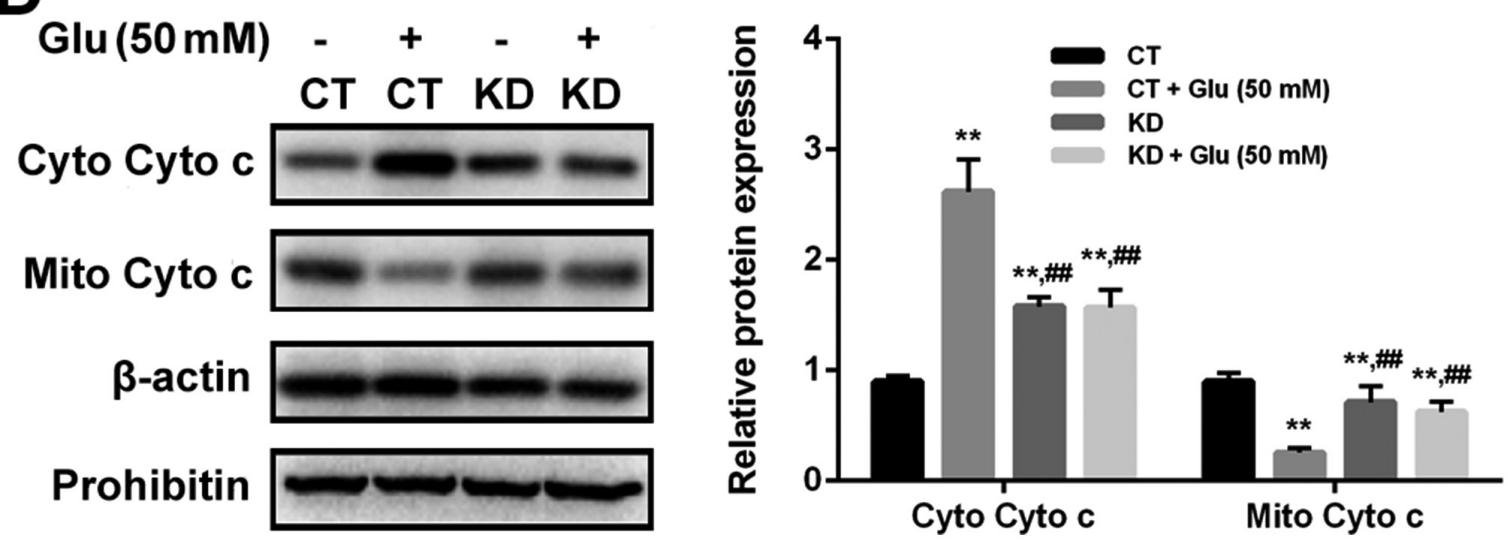

Figure 8. Effect of p66shc knockdown on Glu-induced mitochondrial function in SH-SY5Y cells. Cells with or without p66Shc knockdown were exposed to Glu (50 mM) for 6 h. (A) p66shc knockdown was assessed by western blot analysis. ${ }^{* *} \mathrm{P}<0.01$ vs. scrambled siRNA. (B) MFI of Rh123. (C) Caspase-3 activity. (D) Mito Cyto $c$ and Cyto Cyto $c$ levels were determined by western blot analysis. ${ }^{* *} \mathrm{P}<0.01$ vs. CT; ${ }^{\# \#} \mathrm{P}<0.01$ vs. CT with Glu treatment. Glu, glutamate; GBE, Ginkgo biloba extract; MFI, mean fluorescence intensity; Mito Cyto $c$, mitochondrial cytochrome $c$; Cyto Cyto $c$, cytosolic cytochrome $c$; KD, knockdown; siRNA, small interfering RNA.

alleviate glutamate-induced oxidative toxicity. Consistently, GBE dose-dependently attenuated the oxidative toxicity associated with glutamate through inactivating redoxosome signaling in SH-SY5Y cells. p66Shc, a $66 \mathrm{kDa}$ Src collagen homologue (Shc) adaptor protein, is reported to be downstream effector of redoxosome signaling in several types of cells (33). Genetic deletion of p66Shc adaptor protein prevents hyperglycemia-induced oxidative stress in endothelial cells (35). p66Shc Ser36 phosphorylation induces its translocation to the mitochondrial intermembrane space, enabling its interaction with cytochrome $c$ and promoting the transfer of electrons to oxygen for the generation of hydrogen peroxide and consequently, activating programmed cell death (36). In this study, p66Shc inhibition through siRNA silencing potently prevented cells from the glutamate-induced oxidative toxicity, indicating that p66Shc activation directly contributes to glutamate toxicity in a redoxosome dependent manner. In addition, p66Shc activation reduced MMP, increased the release of cytochrome $c$ and upregulated caspase-3. Interestingly, p66Shc activation and mitochondrial dysfunction can be dose-dependently attenuated 


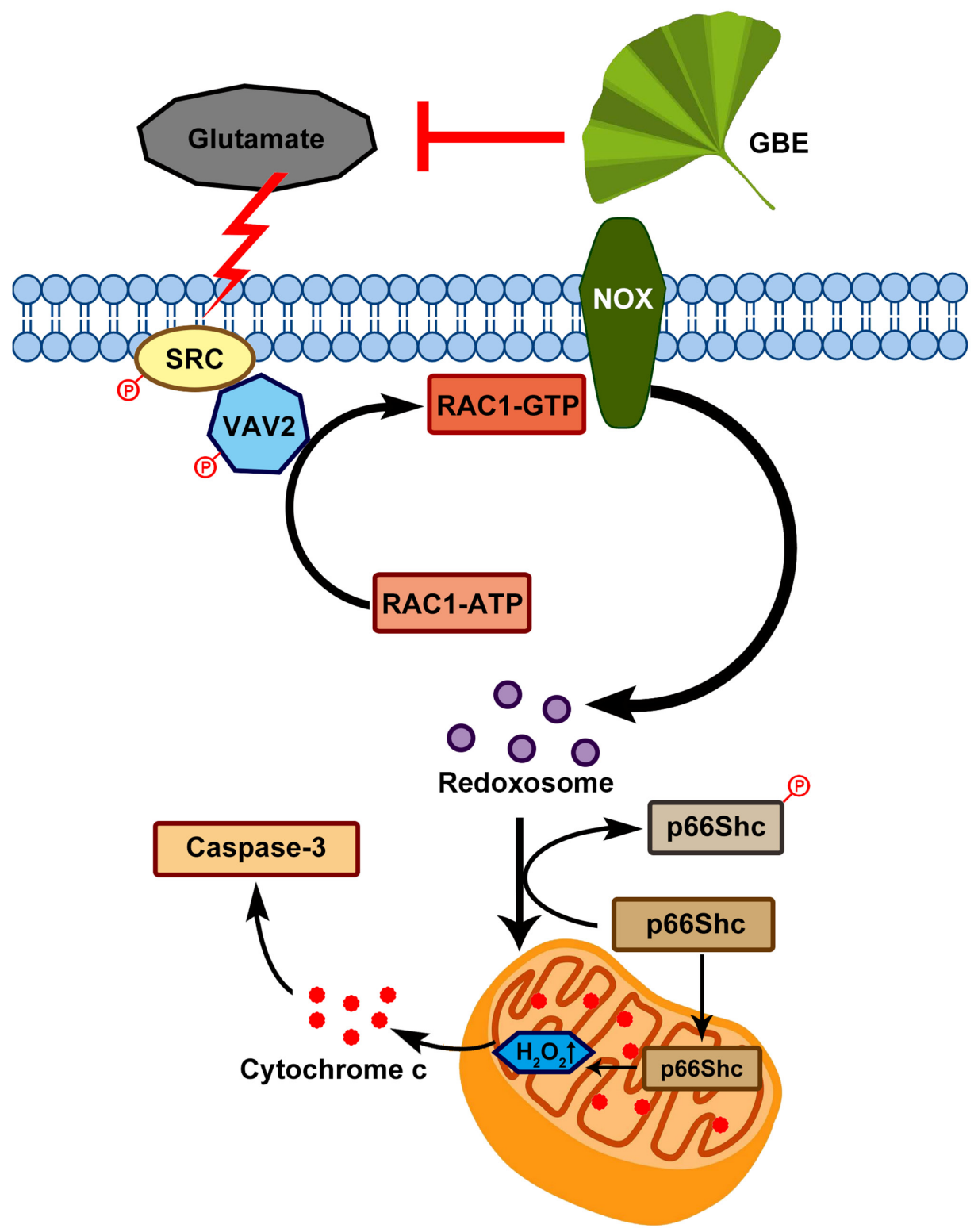

Figure 9. Proposed molecular mechanism underlying the neuroprotective effect of GBE against glutamate-induced oxidative toxicity in SH-SY5Y cells. GBE, Ginkgo biloba extract; SRC, proto-oncogene tyrosine-protein kinase Src; Vav2, guanine nucleotide exchange factor VAV2; P, phosphorylated; NOX, NADPH oxidase.

with the pre-treatment of GBE in SH-SY5Y cells. Our results revealed the novel regulatory mechanism of GBE against glutamate-induced oxidative toxicity.

In conclusion, this study demonstrated the neuroprotective effect of GBE against glutamate-induced oxidative toxicity in SH-SY5Y cells, which effect is possibly mediated through redoxosome-p66Shc signaling (Fig. 9). This provides new information in the pathogenesis of various neurological diseases that are induced by oxidative damage, and also form the basis of the clinical use of GBE in treating these diseases. In addition, this study suggests that redoxosome-p66Shc signaling can be a potential therapeutic target in the prevention/treatment of neurological pathologies.

\section{Acknowledgements}

Not applicable.

\section{Funding}

This work was supported by grants from the Project of National Natural Science Foundation of China (grant no. 81700852), the Young Talent's Subsidy Project in Science and Education of the Department of Public Health of Jiangsu Province (grant no. QNRC2016627), Six Talent Peaks Project in Jiangsu Province (grant no. WSW-047), Six-one Scientific Research Project (grant no. LGY2019087), Innovation Capacity Development Plan of Jiangsu Province (grant no. BM2018023) and the Project of Wuxi Municipal Health Bureau (grant no. Q201813).

\section{Availability of data and materials}

The datasets used and/or analyzed during the current study are available from the corresponding author on reasonable request. 


\section{Authors' contributions}

$\mathrm{KW}$ and $\mathrm{FZ}$ designed the experiments. $\mathrm{KW}, \mathrm{JN}$ and $\mathrm{XZ}$ carried out the experiments. $\mathrm{KW}, \mathrm{JN}$ and $\mathrm{XZ}$ confirmed the authenticity of all the raw data. YL and LZ analyzed the experimental results. KW, FZ and JN wrote the manuscript. All authors read and approved the final manuscript.

\section{Ethics approval and consent to participate}

Not applicable.

\section{Patient consent for publication}

Not applicable.

\section{Competing interests}

The authors declare that they have no competing interests.

\section{References}

1. Hyman SE: Neurotransmitters. Curr Biol 15: R154-R158, 2005.

2. Kanazawa I: Neurotransmitters and neurodegenerative disorders. Clin Ther 7: 48-58, 1984.

3. Shen J: Impaired neurotransmitter release in Alzheimer's and Parkinson's diseases. Neurodegener Dis 7: 80-83, 2010.

4. Meldrum BS: Glutamate as a neurotransmitter in the brain: Review of physiology and pathology. J Nutr 130 (4S Suppl): 1007S-1015S, 2000.

5. Maher P, van Leyen K, Dey PN, Honrath B, Dolga A and Methner A: The role of $\mathrm{Ca}^{2+}$ in cell death caused by oxidative glutamate toxicity and ferroptosis. Cell Calcium 70: 47-55, 2018

6. Lewerenz J and Maher P: Chronic glutamate toxicity in neurodegenerative diseases-what is the evidence? Front Neurosci 9: 469, 2015.

7. Nash KM and Shah ZA: Current perspectives on the beneficial role of Ginkgo biloba in neurological and cerebrovascular disorders. Integr Med Insights 10: 1-9, 2015.

8. Mashayekh A, Pham DL, Yousem DM, Dizon M, Barker PB and Lin DD: Effects of Ginkgo biloba on cerebral blood flow assessed by quantitative MR perfusion imaging: A pilot study. Neuroradiology 53: 185-191, 2011.

9. Ahlemeyer B and Krieglstein J: Neuroprotective effects of Ginkgo biloba extract. Cell Mol Life Sci 60: 1779-1792, 2003.

10. Droy-Lefaix MT: Effect of the antioxidant action of Ginkgo biloba extract (EGb 761) on aging and oxidative stress. Age (Omaha) 20: 141-149, 1997.

11. Park SE, Kim S, Sapkota K and Kim SJ: Neuroprotective effect of rosmarinus officinalis extract on human dopaminergic cell line, SH-SY5Y. Cell Mol Neurobiol 30: 759-767, 2010.

12. Gismondi A, Trionfera E, Canuti L, Di Marco G and Canini A: Royal jelly lipophilic fraction induces antiproliferative effects on SH-SY5Y human neuroblastoma cells. Oncol Rep 38: 1833-1844, 2017.

13. Lantto TA, Colucci M,Závadová V, Hiltunen R and Raasmaja A: Cytotoxicity of curcumin, resveratrol and plant extracts from basil, juniper, laurel and parsley in SH-SY5Y and CV1-P cells. Food Chem 117: 405-411, 2009.

14. Rahman MA, Yang H, Lim SS and Huh SO: Apoptotic effects of melandryum firmum root extracts in human SH-SY5Y neuroblastoma cells. Exp Neurobiol 22: 208-213, 2013.

15. Izuta H, Shimazawa M, Tazawa S, Araki Y, Mishima S and Hara H: Protective effects of Chinese propolis and its component, chrysin, against neuronal cell death via inhibition of mitochondrial apoptosis pathway in SH-SY5Y cells. J Agric Food Chem 56: 8944-8953, 2008.

16. Zhu X, Xue L, Yao Y, Wang K, Tan C, Zhuang M, Zhou F and Zhu L: The FoxM1-ABCC4 axis mediates carboplatin resistance in human retinoblastoma Y-79 cells. Acta Biochim Biophys Sin (Shanghai) 50: 914-920, 2018.
17. Zhu P, Tong BM, Wang R, Chen JP, Foo S, Chong HC, Wang XL, Ang GY, Chiba S and Tan NS: Nox4-dependent ROS modulation by amino endoperoxides to induce apoptosis in cancer cells. Cell Death Dis 4: e552, 2013

18. Karamitros CS, Lim J and Konrad M: An amplex red-based fluorometric and spectrophotometric assay for L-asparaginase using its natural substrate. Anal Biochem 445: 20-23, 2014.

19. Wang Y and Lou MF: The regulation of NADPH oxidase and its association with cell proliferation in human lens epithelial cells. Invest Ophthalmol Vis Sci 50: 2291-2300, 2009.

20. Yang J, Liu X, Bhalla K, Kim CN, Ibrado AM, Cai J, Peng TI, Jones DP and Wang X: Prevention of apoptosis by Bcl-2: Release of cytochrome c from mitochondria blocked. Science 275 : 1129-1132, 1997.

21. Christen Y: Ginkgo biloba and neurodegenerative disorders. Front Biosci 9: 3091-3104, 2004.

22. Bastianetto S, Ramassamy C, Doré S, Christen Y, Poirier J and Quirion R: The Ginkgo biloba extract (EGb 761) protects hippocampal neurons against cell death induced by beta-amyloid. Eur J Neurosci 12: 1882-1890, 2000.

23. Müller WE, Eckert A, Eckert GP, Fink H, Friedland K, Gauthier S, Hoerr R, Ihl R, Kasper S and Möller HJ: Therapeutic efficacy of the Ginkgo special extract EGb761 ${ }^{\circledR}$ within the framework of the mitochondrial cascade hypothesis of Alzheimer's disease. World J Biol Psychiatry 20: 173-189, 2019.

24. Ren C, Ji YQ, Liu H, Wang Z, Wang JH, Zhang CY, Guan LN and Yin PY: Effects of Ginkgo biloba extract EGb761 on neural differentiation of stem cells offer new hope for neurological disease treatment. Neural Regen Res 14: 1152-1157, 2019.

25. Yao ZX, Han Z, Drieu K and Papadopoulos V: Ginkgo biloba extract (Egb 761) inhibits beta-amyloid production by lowering free cholesterol levels. J Nutr Biochem 15: 749-756, 2004.

26. Huang DS, Lin HY, Lee-Chen GJ, Hsieh-Li HM, Wu CH and Lin JY: Treatment with a Ginkgo biloba extract, EGb 761, inhibits excitotoxicity in an animal model of spinocerebellar ataxia type 17. Drug Des Devel Ther 10: 723-731, 2016.

27. Lee HJ, Spandidos DA, Tsatsakis A, Margina D, Izotov BN and Yang SH: Neuroprotective effects of scrophularia buergeriana extract against glutamate-induced toxicity in SH-SY5Y cells. Int J Mol Med 43: 2144-2152, 2019.

28. Sun X, Shi X, Lu L, Jiang Y and Liu B: Stimulus-dependent neuronal cell responses in SH-SY5Y neuroblastoma cells. Mol Med Rep 13: 2215-2220, 2016.

29. Ba XH and Min LQ: Effects of Ginkgo biloba extract on the apoptosis of oxygen and glucose-deprived SH-SY5Y cells and its mechanism. Indian J Pharmacol 47: 101-104, 2015.

30. Bekeschus S, Bräutigam L, Wende K and Hanschmann EM: Oxidants and redox signaling: Perspectives in cancer therapy, inflammation, and plasma medicine. Oxid Med Cell Longev 2017: 4020253, 2017.

31. Hsieh HL and Yang CM: Role of redox signaling in neuroinflammation and neurodegenerative diseases. Biomed Res Int 2013: 484613, 2013.

32. Franco $R$ and Vargas MR: Redox biology in neurological function, dysfunction, and aging. Antioxid Redox Signal 28: 1583-1586, 2018.

33. Karunakaran U, Elumalai S, Moon JS and Won KC: CD36 dependent redoxosomes promotes ceramide-mediated pancreatic $\beta$-cell failure via p66Shc activation. Free Radic Biol Med 134: 505-515, 2019.

34. Spencer NY and Engelhardt JF: The basic biology of redoxosomes in cytokine-mediated signal transduction and implications for disease-specific therapies. Biochemistry 53: 1551-1564, 2014.

35. Kumar S: P66Shc and vascular endothelial function. Biosci Rep 39: BSR20182134, 2019.

36. Zhang M, Tang J, Shan H, Zhang Q, Yang X, Zhang J and Li Y: P66Shc mediates mitochondrial dysfunction dependent on PKC activation in airway epithelial cells induced by cigarette smoke. Oxid Med Cell Longev 2018: 5837123, 2018.

This work is licensed under a Creative Commons Attribution-NonCommercial-NoDerivatives 4.0 International (CC BY-NC-ND 4.0) License. 